\title{
THERMODYNAMIC INTERACTION OF THE PRIMARY PROTON BEAM WITH A MERCURY JET TARGET AT A NEUTRINO FACTORY SOURCE*
}

\author{
N. Simos ${ }^{\dagger}$, H. Ludewig, H. Kirk, P. Thieberger, BNL, Upton, NY 11793, USA \\ K. McDonald, Princeton University, Princeton, NJ 08544, USA
}

\begin{abstract}
This paper addresses the thermodynamic interaction of an intense proton beam with the proposed mercury jet target at a neutrino factory or muon collider source, and the consequences of the generated pressure waves on the target integrity. Specifically, a $24 \mathrm{GeV}$ proton beam with approximately $1.6 \mathrm{e} 13$ protons per pulse and a pulse length of 2 nanosec will interact with a $1 \mathrm{~cm}$ diameter mercury jet within a 20 Tesla magnetic field. In one option, a train of six such proton pulses is to be delivered on target within 2 microsec, in which case the state of the mercury jet following the interaction with each pulse is critical. Using the equation of state for mercury from the SESAME library, in combination with the energy deposition rates calculated the by the hadron interaction code MARS, the induced 3-D pressure field in the target is estimated. The consequent pressure wave propagation and attenuation in the mercury jet is calculated using an ANSYS code transient analysis, and the state of the mercury jet at the time of arrival of the subsequent pulse is assessed. The amplitude of the pressure wave reaching the nozzle that ejects the mercury jet into the magnetic field is estimated and the potential for mechanical damage is addressed.
\end{abstract}

\section{INTRODUCTION}

A challenging design issue within the target space of a muon collider is the possibility of shock wave impact and consequently potential damage on the jet nozzle.

The proposed target is a $1 \mathrm{~cm}$ diameter mercury jet ejected from a nozzle into a 20 Tesla magnetic field where it is intercepted by a $24 \mathrm{GeV}$, tightly focused proton beam with $0.5 \mathrm{~mm}$ rms sigma and intensity of 16 TP. A train of six 2 ns long pulses represents one of the options. The proton intensity of each micro-pulse combined with the short pulse length is bound to induce very high pressures in the mercury. While the major issue is the likelihood of jet destruction from a single micropulse, the potential for pressure waves arriving at the nozzle is of engineering importance. These pressure waves are generated in the $\mathrm{Hg}$ jet from its interaction with the proton beam and travel back toward the nozzle along the continuous jet.

\subsection{Thermodynamic Interaction}

The energy deposited in the $\mathrm{Hg}$ jet has bee calculated using MARS [4]. The first set of deposition calculations considered the proton pulse and the jet to meet head on. According to that scenario the peak energy of 87 Joules/gram appears at about $5 \mathrm{~cm}$ into the jet. The results presented in this paper are based on these results. In the latest option the jet is tilted by $100 \mathrm{mrad}$ and the proton beam by $67 \mathrm{mrad}$. A schematic is shown in Figure 1. Based on the latest calculations, the peak energy deposited is about 50 Joules/gram.

While the initial temperature and pressure can be estimated using approximate formulae $(\Delta \mathrm{Q}=\mathrm{c} \Delta \mathrm{T}$ and $\Delta \mathrm{P}=\alpha_{\mathrm{v}} \Delta \mathrm{T} / \kappa$ respectively, where $\kappa$ is the compressibility and $\alpha_{\mathrm{v}}$ is the expansion coefficient for $\mathrm{Hg}$ ), the thermodynamic processes that take place between pulses need the equation of state for mercury in order to be traced properly. The SESAME library for $\mathrm{Hg}$ provides such a relationship and is used to assess the pressure and temperature resulting from a series of micropulses. Specifically, prior to its interaction with the beam, the jet is in a compressed liquid state. At the end of the short pulse, the mercury has increased its pressure and temperature through constant volume process but is still a highly compressed liquid. What follows is an adiabatic expansion of the pressurized zone (adiabatic because heat transfer is much too slow a process to take place at these small times). The heated and pressurized $\mathrm{Hg}$ expands pushing the surrounding mercury. The volumetric change of the heated zone is proportional to the stretching of the interface. Estimates of such volumetric change have been made that led to the approximate formula for the outward velocity $\mathrm{V}=\sqrt{ } 2 \alpha_{\mathrm{v}} \Delta \mathrm{Tc}$, where $\mathrm{c}$ is the sound velocity. Along with the volumetric expansion, and due to pressure gradients, pressure waves are generated and propagate through the mercury.

Based on the original interaction model and the use of the SESAME equation of state for thermodynamic processes, it is estimated that the mercury may enter the critical zone after the third micropulse. It is possible that with the later jet, beam arrangement with lower energy deposition that the jet may survive more pulses.

\footnotetext{
* Work performed under the auspices of the US DOE

†simos@bnl.gov
} 


\subsection{Pressure Wave/Nozzle Interaction}

The initial pressures that are generated in the interaction zone of the jet are approaching $3800 \mathrm{MPa}$. While the interaction zone of the jet may be broken up a few microseconds after the proton beam arrival, the upstream section of the jet is still intact and will allow for the propagation of pressure waves toward the nozzle. At issue is the amplitude of the pressure wave front when it arrives at the nozzle and impacts on the walls. The estimated time of the arrival of the front is approximately 100 micro-secs based on a $15 \mathrm{~cm}$ distance between the beginning of the interaction zone and the nozzle. Figure 1 shows the schematic of the model that was used.

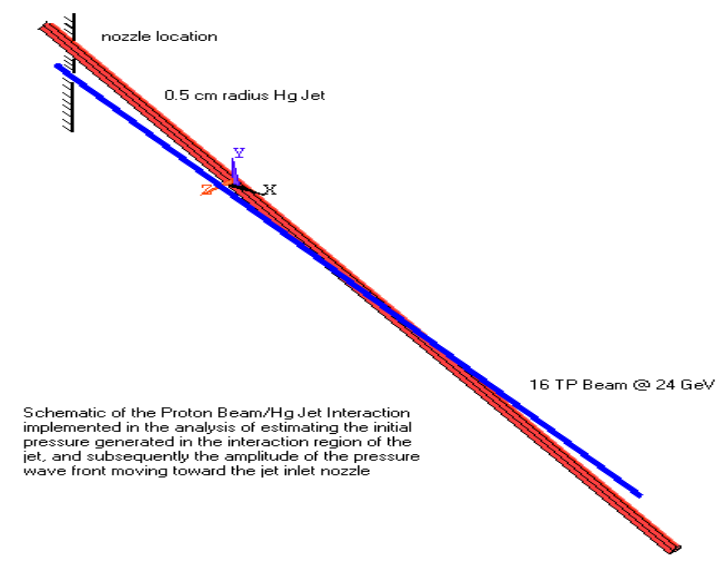

Figure 1: Schematic of the Hg Jet/Proton Beam Interaction implemented in the pressure wave analysis

Figures 2 through 4 below show snapshots of the pressure profile along the $\mathrm{Hg}$ jet in a cut through the long axis. While pressures start out as positive as a result of the rapid energy deposition and the inability of the $\mathrm{Hg}$ to accommodate the thermal expansion, it quickly turns negative in the center of the interaction zone as a result of the wave reflections and sign reversal from the free surface of the jet. As mentioned above, while part of the interaction region may be destroyed, the pressure front will advance toward the nozzle.

As expected, the pressure wave will attenuate as it travels through the undisturbed part of the jet. Figure 5 depict the pressure wave fluctuation and amplitude at different in the nozzle vicinity. The amplitude of the pressure wave when it arrives at the nozzle is approximately $100 \mathrm{MPa}$. While such a pressure may result in nozzle and jet channel stresses that are below the strength limits, a large number of such impacts will accumulate during the operation of the machine that may lead to fatigue failure. The latter becomes more of an issue considering the high irradiation doses the structural materials will receive because of their proximity to the target.

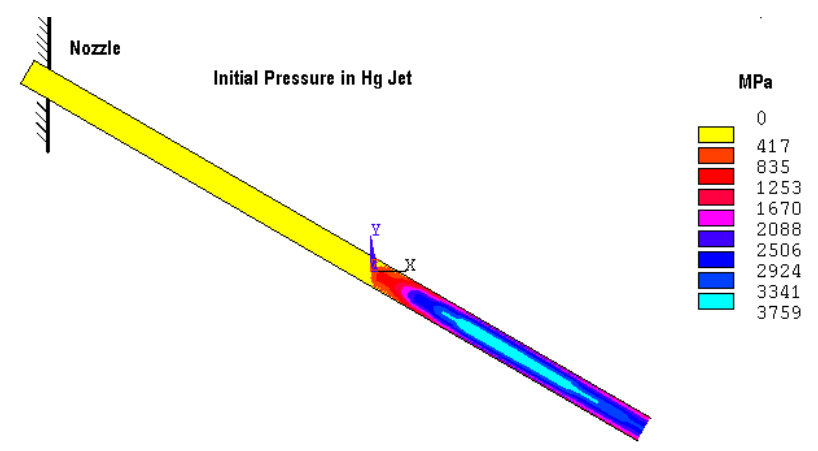

Figure 2: Initial Pressure in the $\mathrm{Hg}$ Jet

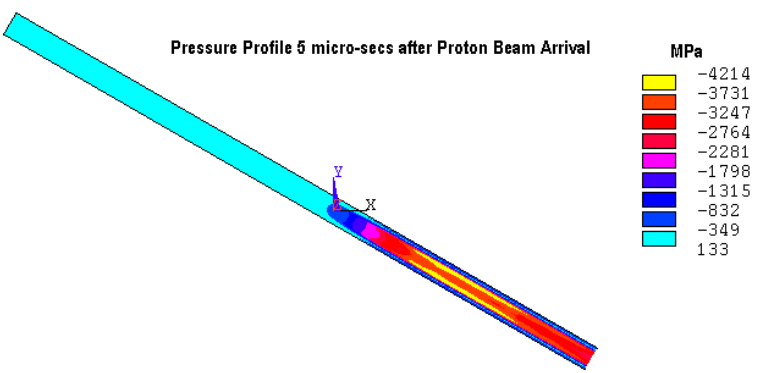

Figure 3: Pressure profile $5 \mu$ s after beam arrival
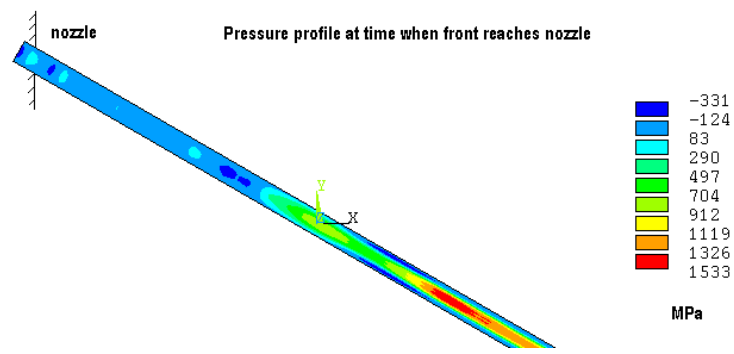

Figure 4: Pressure profile upon arrival of the front at nozzle location

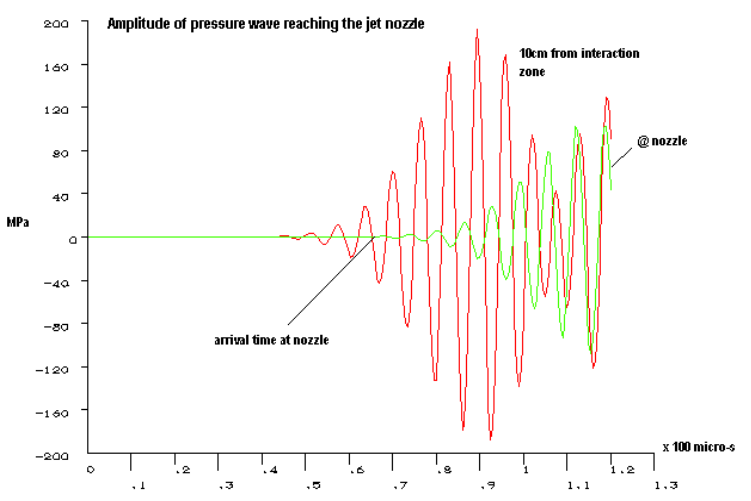

Figure 5: Pressure wave amplitude arriving at nozzle 


\section{E951 EXPERIMENT AT BNL}

In the BNL muon targetry experiment E951 the interaction of an $\mathrm{Hg}$ jet with a proton beam was studied without the 20 Tesla magnetic field. A schematic of the target chamber is shown in Figure 7. The available setup provided an opportunity to address the issue of shock waves reaching the jet nozzle. As a result, four fiber optic strain gauges were placed at selected locations in the mercury line.

These locations are shown in the details of Figure 6. Specifically, a gauge was placed on the line that supplies mercury to the jet just upstream of the nozzle. This gauge, placed along the pipe as shown, is expected to register any activity associated with a wave returning from the jet. The geometry and size of the supply pipe did not allow for the gauge to be placed with hoop orientation. Potential strains along the hoop direction in the pipe wall are expected to be much higher than the axial and thus more easily detectable. Strains in the supply pipe will be the direct result of the pressure in the contained mercury.

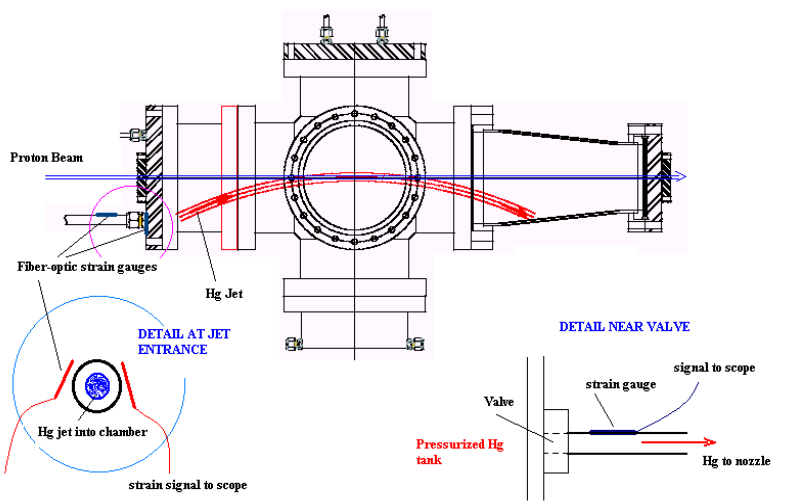

Figure 6. Schematic of the E951 mercury target set-up and strain gauge array

In addition to the nozzle gauge, one was placed at the valve outlet (furthest location in the supply pipe upstream of the nozzle) and two were installed on the nozzle mounting plate on either side of the nozzle.

An extensive set of strain measurements were collected for beam intensities ranging between $0 \mathrm{TP}$ and $4 \mathrm{TP}(0$ $\mathrm{TP}$ being the case of jet activity alone). While the beam intensity was much lower than anticipated, thus keeping the potential strain aggravation due to shock quite low, still some clear evidence of activity was recorded. Shown in Figure 7 is the strain recorded by the nozzle gauge for back to back pulses with similar intensities (3.75 TP). The stability in the measuring system is shown to be excellent. The front part of the record is the noise from the flowing $\mathrm{Hg}$ in the supply pipe. The spike indicates the arrival of the proton beam and it is the effect of photons on the gauges. Beyond that there is clear evidence of activity induced by the proton beam interacting with the jet. Figure 8 quantifies the effect by comparing the strain induced by the jet alone with that of the interaction. Not shown here is the dramatic effect induced by the operation of the valve that initiates the jet and especially its closing where the "hammer" action is dominant.

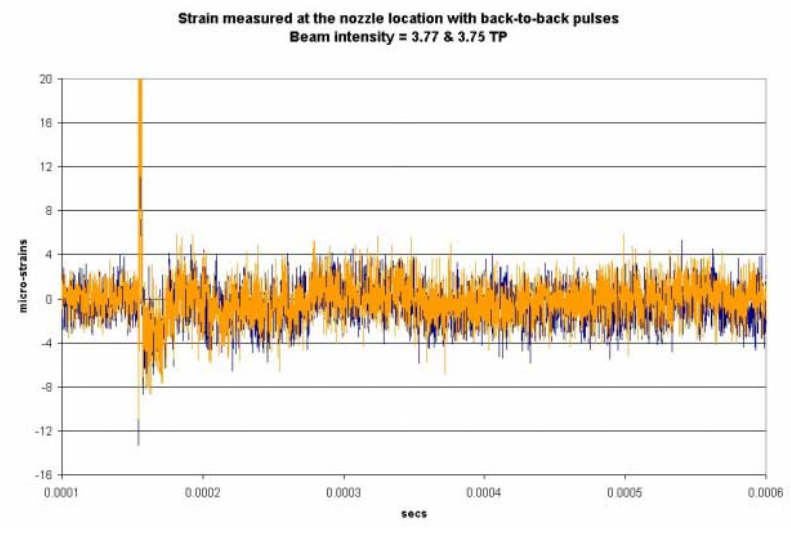

Figure 7. Strain near nozzle from back to back pulses

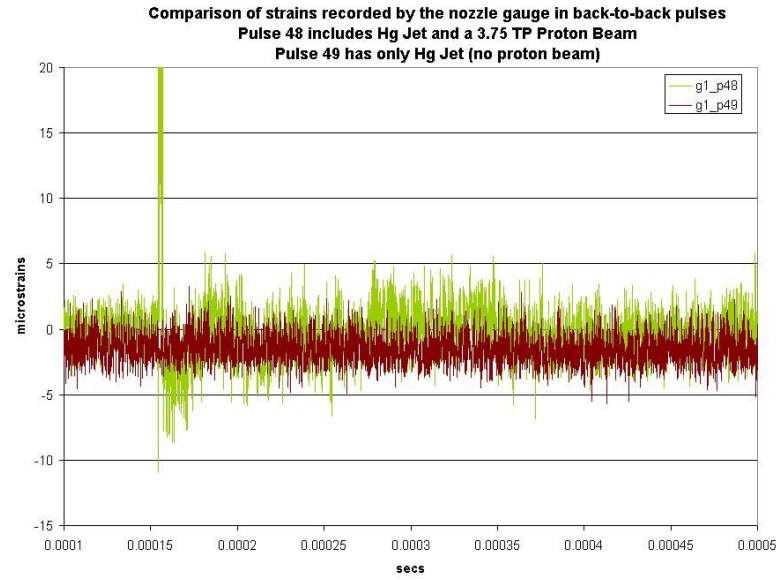

Figure 8. Strain comparison between the case of jet and beam interaction and jet only

\section{REFERENCES}

[ 1 ]P. Sivers and P. Pugnat, " Response of Solid and Liquid Targets to High Power Proton Beams for Neutrino Factories,"

[ 2 ] SESAME Library, SANL

[ 3 ]V. W. Sonntag, "Fundamentals of Classical Thermodynamics", $2^{\text {nd }}$ Edition, Wiley, 1973

[ 4 ]N.V. Mokhov, "The MARS Code System User Guide, Version 13 (95)", 1995

[ 5 ]N. Molkov, Private communication

[6]ANSYS Engineering Analysis of Systems, Swanson Analysis Systems Inc., 1999 